Año LXXXII. urtea $279-2021$ Enero-abril Urtarrila-apirila

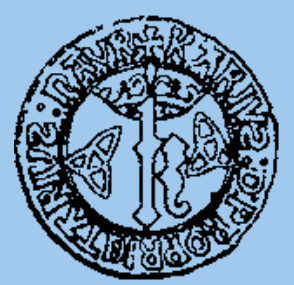

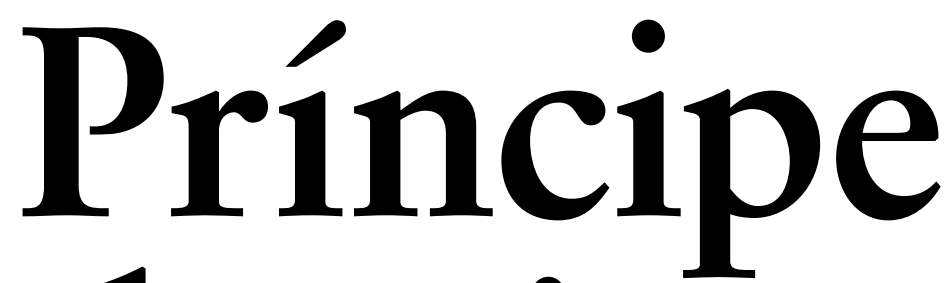

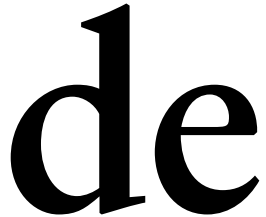
$\mathrm{Vi}$ iana

SEPARATA

\section{El alivio y reparo.} La forma audiovisual

Marga GUTIÉRREZ DÍEZ 


\section{Sumario / Aurkibidea}

\section{Príncipe de Viana}

Año LXXXII • n. ${ }^{\circ} 279$ - enero-abril de 2021

LXXXII. urtea $\cdot 279$. zk. 2021 ko urtarrila-apirila

\section{GUSTAV HENNINGSEN / MARISA REY-HENNINGSEN}

\section{Homenaje / Omenaldia}

Ignacio Panizo (coord./koord.)

Vol. II. lib.

\section{ESTUDIOS DE INVESTIGACIÓN EN HOMENAJE \\ A GUSTAV HENNINGSEN Y MARISA REY-HENNINGSEN / \\ IKERKETA LANAK, GUSTAV HENNINGSENEN \\ ETA MARISA REY-HENNINGSENEN OMENEZKOAK}

Entre impostores anda el juego en el Siglo de Oro. Dos vizcaínos ante la Inquisición y unos conversos toledanos próximos a Garibay

Un formulario de la Secretaría de Aragón del Consejo de la Inquisición (BC, ms. 2168)

Anna Gudayol

Las cartillas editadas en Estella e incautadas en Medellín por la Inquisición (1561)

Javier Itúrbide Díaz

El Tribunal de Navarra y sus funcionarios inquisitoriales:

algunas observaciones

Consuelo Juanto Jiménez

Goya investigado por la Inquisición: la censura de los Caprichos en 1804

$\mathrm{El}$ «Informe del inquisidor Avellaneda» sobre las complicidades brujeriles en los valles centrales y orientales del Pirineo navarro José M. Floristán Imízcoz, Jesús Moya Mangas 


\section{Sumario / Aurkibidea}

La Inquisición en Tudela durante los siglos XVI y XVII:

presencia e incidencia social

Iñigo Pérez Ochoa

La huella de la Inquisición en la Biblioteca de Navarra

Roberto San Martín Casi

Los memoriales como fuente para el estudio de la Inquisición

en el Tribunal de Navarra (1609-1621)

Bárbara Santiago Medina

De comisarios, confesores y vecinos: la proyección de la Inquisición

a finales del Antiguo Régimen

Marina Torres Arce

Los niños como víctimas y los niños como verdugos en la caza de brujas:

Navarra, siglo XVI

Jesús M. Usunáriz

Volver a las relaciones de causas. El ejemplo de los berberiscos

del reino de Granada

Bernard Vincent

\section{LOS TRABAJOS Y LOS DÍAS DEL AÑO 2020 / 2020ko LANAK ETA EGUNAK}

Tesis doctorales sobre temática navarra de ciencias humanas, sociales y jurídicas, leídas en 2020

(Según la Base de datos Teseo del Ministerio de Educación)

Narrativa de autores navarros en castellano, año 2020

Mikel Zuza Viniegra

Euskarazko literaturak 2020an zer?

Ángel Erro Jiménez

Donación del fondo bibliográfico y documental Gustav Henningsen y Marisa

Rey a la Universidad Pública de Navarra

Iñaki Montoya Ortigosa

Formación en Archivística y Gestión Documental en la Universidad de Navarra

Yolanda Cagigas Ocejo

6 Príncipe de Viana (PV), 279, urtarrila-apirila, 2021

ISSN: 0032-8472 | ISSN-e: 2530-5824 | ISSN-L: 0032-8472

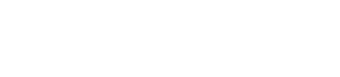




\section{Sumario / Aurkibidea}

Apuntes sobre etnografía, folclore y cultura tradicional David Mariezkurrena Iturmendi

El alivio y reparo. La forma audiovisual

Marga Gutiérrez Díez

Proceso de decantación. Exponer en 2020

Mireya Martín Larumbe

Exposición «Maleficium. Navarra y la caza de brujas. Siglos XIV-XVII»

(Archivo Real y General de Navarra, julio-diciembre, 2020)

Jesús M. Usunáriz

Carlos Cánovas

Alicia Ezker Calvo

Discurso Premio Príncipe de Viana 2020

Carlos Cánovas Ciaurriz

Currículums

Analytic Summary

Normas para la presentación de originales / Idazlanak aurkezteko arauak /

Rules for the submission of originals 


\title{
El alivio y reparo. La forma audiovisual
}

\author{
Marga Gutiérrez Díez \\ Gerente del CLAVNA (febrero de 2017 a marzo de 2018) \\ marga@laescenadeljardin.com
}

DOI: https://doi.org/10.35462/pv.279.19
El año que es abundante de poesía, suele serlo de hambre.
Los trabajos de Persiles
y Sigismunda, historia setentrional
Última obra Miguel de Cervantes

Muchas veces repite Cervantes en sus obras que el poeta es siempre pobre, quizá una triste nota autobiográfica: «Pues cierto que es más milagro darme a mí un poeta un escudo, que yo recibirle» (La gitanilla). Situación que, muy a nuestro pesar, abordan muchas profesiones de las bellas artes. Artistas, técnicos, músicos y empresas colindantes han sufrido de lleno un parón casi total. Y donde el audiovisual en su faceta de producción de contenidos se ha salvado y en algunos casos ha solventado con ánimo y productividad en el año de la pandemia.

Un año para parar; y «el que para repara», dice el dicho castellano. Un enfoque para comenzar un artículo que a priori parece más sencillo si abordamos el audiovisual del 2020 con perspectiva de menos hitos, menos producciones, menos formas de contar y relatar historias. Carencias que no fueron otra cosa que una catapulta para el consumo en los hogares de todas las formas posibles de lo visual: desde conexiones online que saturaban la red hasta plataformas con suscripciones que han batido record. Todo muy virtual. Todo muy diferente.

Para ubicar el año, el $45 \%$ de profesionales del sector audiovisual de Navarra hablan de pérdidas de hasta el $90 \%$, dato que se extrae de la encuesta realizada por el Clúster 
Audiovisual de Navarra: el 44,7\% de los 88 encuestados, valoraba que tendría unas pérdidas de entre un $50 \%$ y un $90 \%$. El $27,1 \%$ estimaba que esas pérdidas serían del $100 \%$ a corto plazo y un $8,1 \%$ había cancelado proyectos definitivamente. Muchas de las productoras y técnicos se han agarrado a proyectos más institucionales, comerciales y específicos de empresa privada para generar contenidos también que se adaptaran al cambio.

Solamente una vez este artículo va a nombrar la palabra y nomenclatura que atañe y empaña el año veinte del siglo XXI, un año de resiliencia, de afrontar cambios, de mirar de otro modo y de supervivencia de empresas y profesionales audiovisuales navarros que no son héroes ni heroínas, sino trabajadores, que como tantos otros han sopesado y apostado por la profesión. Y es que como decía el cantante flamenco Camarón de la Isla cuando le preguntaban por qué cantaba flamenco, su respuesta era clara "no sé hacer mejor otra cosa».

\section{PLAN REACTIVAR NAVARRA}

Las industrias creativas han entrado en el foco de prioridades dentro de este Plan, cuyo Comité de Dirección, presidía el consejero Manu Ayerdi, con la participación del consejero Juan Cruz Cigudosa y la asistencia de clústeres, las universidades, centros tecnológicos, organizaciones empresariales, sindicales y de diversas empresas de referencia para generar la hoja de ruta y dar forma a retos futuros.

Los encuentros y reuniones para reivindicar su espacio han venido marcados por declaraciones institucionales, como las de Izaskun Goñi, directora general de Política Empresarial, Proyección Internacional y Trabajo del Gobierno de Navarra: «El sector audiovisual es una de las actividades emergentes dentro de la Estrategia de Especialización Inteligente de Navarra».

Fiel reflejo de este interés se expuso en el espacio de debate «El audiovisual como sector esencial: La excepción navarra» que Goñi compartió con Sara Sevilla (Navarra Film Comission), Arturo Cisneros (Clúster Audiovisual de Navarra) y Julio Mazarico (Asociación de Productoras y Profesionales del Audiovisual en Navarra), donde se destacó el incentivo fiscal aplicado a estos proyectos que se traduce en un $35 \%$ de desgravación en el Impuesto de Sociedades.

Clavna presentó una lista de destacadas propuestas a corto plazo como:

- Incremento de 5 puntos del incentivo fiscal y 10 para la Animación. Así como la activación de la prevalidación de incentivo.

- Igualar límites de intensidad de ayudas de territorio común y ayudas para adaptación de empresas.

- Apoyar el proyecto Bélgica-Navarra y diseño del fondo Sodena Animation. 
Con vista al futuro se puede destacar su petición de aumentar apoyos a la política I+D+i puesta en marcha desde CLAVNA; abrir líneas específicas de I+D para empresas audiovisuales de cuantías de entre 50000 y 200000 euros y activar un convenio Navarra-Euskadi-Aquitania (Eurociduda) como destino de cine colaborativo

\section{GENERAZINEMA 2020}

Como es ya cíclico y más que fundamental, el Departamento de Cultura y Deporte ha concedido 598592 euros en ayudas al sector audiovisual de Navarra. Se incrementaron las ayudas en 207000 euros respecto a 2019. Destaca que se van adaptando a la realidad cinematográfica, que alivian y facilitan los procesos: ayuda al desarrollo de forma plurianual; una ayuda especial para proyectos de bajo presupuesto; rebaja del porcentaje de financiación previa necesaria en producción y en desarrollo.

Cinco convocatorias Generazinema 2020 financiaron veinte películas y se diferenciaron, por primera vez, con ayudas específicas para la fase de producción y fase de desarrollo de largometrajes y cortometrajes:

Las ayudas a la producción ascienden a 229110 euros entre cuatro largometrajes. Se trata de la película de ficción Espárrago, de Julio Mazarico, que recibe 110000 euros, y de los documentales Ainarak, de 601 Producciones Audiovisuales y Zinea Sortzen, dirigido por Juan San Martín, que obtiene una subvención de 60000 euros; Dardara, de Arena Comunicación, de Marina Lameiro, con 39.160 euros; y Non dago Mikel?, de Izar Films, dirigido por Amaia Merino y Miguel Ángel Llamas (19950 euros).

En cortometrajes se repartieron entre Bizimina, de Arena Comunicación (25000 euros), dirigida por Pablo Iraburu y Migueltxo Molina; Unicornio, de Maddi Barber (12 090 euros), y los cortos de animación ¿Dónde está Heleny?, de Tripulante Produce (28 000 euros) y Cafuné, de The New gravity laws (20 795 euros).

En la fase de desarrollo la ayuda se distribuye entre Feliz final, de Lamia Producciones (20500 euros), que dirigirá Helena Taberna; Nina, de Lasai Producciones, de Andrea Jaurrieta (20 500 euros); Censurada, de Koolshiit Productions, dirigida por Sarah Gamazo y Mario Garza (17058 euros); y Modu modua, de Goizeder Urtasun (7567 euros).

Los documentales también tuvieron cabida: Volver, de Arena Comunicación (20 500 euros), que dirigirán Pablo Iraburu y Migueltxo Molina; Petrus, de Solax Company, dirigido por Helena Bengoetxea (15642 euros); La profecía, de Maracama Films, dirigido por Amaia Remírez y Raúl de la Fuente (9500 euros); Black Water, de Kanaki Films, dirigido por Natxo Leuza (7754 euros); y Éramos, de Hiruki Films, de Marina Lameiro (7600 euros). Asimismo, se otorga ayuda para el desarrollo de los largometrajes de animación Evolution, de Viuda Negra y The Think Lab Media (36250 euros); Dinogames, de Kakuru-Sam (30 500 euros) y Ojalá, de Demiranda Studio/NASA (25889 euros). 
En Festivales se otorgaron 10000 euros de ayuda para el Festival NIFF 2020; al FAN 2020 se le conceden 9000 euros. Del mismo modo, al Certamen Navarra, Tierra de cine se le asignan 8973 euros; para el VII edición del Concurso CTL 59 segundos se conceden 702 euros y para Uda Golem Verano 2020 se ha aprobado una ayuda de 8760 euros.

En la línea nueva de subvenciones para la exhibición de cine europeo e iberoamericano (Generazinema exhibición 2020) han obtenido 5000 euros de ayudas cada una de las cuatro empresas solicitantes: Areto, S.L. (responsable de las salas de cine de Estella), Pedro José Sagardoy Alli (para la sala de cine de Tafalla), Difusora Cultural Cinematográfica, S.A. Yamaguchi y Difusora Cultural Cinematográfica S. A Baiona.

Y para Generazinema Exporta, para profesionales en festivales, mercados, ferias y eventos profesionales de cinematografía y audiovisual: Kanaki Films (2921 euros), Golem Distribución (3000 euros) y Mikel Xabier Arraiz Aldunate (981,58 euros).

\section{NAPAR, ASOCIACIÓN NAVARRA DE PRODUCTORAS}

La asociación de productora de Navarra cuenta ya con 46 socios.

Entre las actividades a destacar se encuentran los «Hablamos con... encuentros profesionales con la industria» que se realizaron con Garbiñe Ortega, directora artística del Festival Internacional de Cine Documental Punto de Vista; Lois Patiño, realizador de cine y la proyección de su película Costa da norte; Pedro Martín, es actual director del Área Audiovisual, Cultura y Deporte de CREA SGR, entidad financiera de ámbito nacional; Andrés Luque Pérez, productor ejecutivo de coproducción Documentales de La 2; Sergio Villava, actual gerente de Consorcio Eder.

Desarrollaron actividades profesionales en el marco de algunos festivales como Punto de Vista, NIFF, Zinetika, SSIFF o Navarra Tierra de Cine y el ciclo en Filmoteca, de Napar. Además, por la naturaleza del sector y su relación con los derechos, NAPAR se ha vinculado con INTANGIA para poder dar a los socios y socias de NAPAR un servicio de amparo y consejo legal en esta materia. Y forma parte de la actual Federación PIAF (Productoras Independientes Audiovisuales Federadas).

\section{NAVARRA FILM COMMISSION NFC}

Desde la oficina que gestiona los proyectos y el impulso del sector audiovisual navarro se ha trabajado de forma conjunta entre Spain Film Commission y las diferentes comunidades en elaborar los documentos necesarios para la reactivación y buena consecución de la actividad audiovisual en el país. Con el trabajo en conjunto del Departamento de Cultura y Deporte de Gobierno de Navarra y Napar -Asociación de Productoras Audiovisuales de Navarra- para el manual de buenas prácticas -ante la pandemia- como guía del sector. 
Navarra Film Commission ha iniciado colaboraciones y convenios con diferentes organizaciones como son Nasuvinsa, el Departamento de Desarrollo Económico y el de Desarrollo Rural y Medio Ambiente del Gobierno de Navarra así como con la Universidad de Navarra; los objetivos que se persiguen son la promoción del territorio, del turismo y velar por la industria cinematográfica, con una mirada transversal entre sectores.

NFC ha estado en el Certamen de Cortometrajes Navarra Tierra de Cine, NIFF (Navarra International Film Festival), Lo que viene, Conecta Fiction, Zinetika. Y no pudo acudir, al ser cancelados, a: Skimetraje, CTL 59, Opera Prima, Gala concurso SciencEkaitza

Junto a la Asociación de productores de Navarra (NAPAR y el Cluster Audiovisual Navarro (CLAVNA) sus acciones fueron ligadas a:

- Participación en las mesas técnicas: grupos mixtos de socios y colaboradores para dar forma al plan estratégico.

- Comunicación de la información relevante del sector (Newsletter).

- Guía del AV Navarro:

- Guía con toda la información necesaria de recursos tanto humanos como técnicos alrededor de la producción.

- Pedagogía de la deducción fiscal a la producción normativa de deducción fiscal para los proyectos audiovisuales.

- Búsqueda de nuevos socios.

- Ampliación a 36 socios.

- Hub Audiovisual de Navarra.

Las ferias y foros industriales a los que se ha asistido como miembro acreditado: FITUR, European Film Market, Berlín, Conecta Fiction, Pamplona, Zinemaldi. Ha participado en acciones suspendidas o celebradas de forma online en las siguientes ferias: Series Mania, Lille (Pospuesto 2021), MAFIZ, Málaga (Pospuesto online), Mestizo Lab Foro Coproducción España/México, Seminci, Valladolid, Hendaia Film Festival (Pospuesto2021), Series Nostrum, Altea.

Se mantienen las relaciones como socio de Spain Film Commission (SFC). Desde el mes de diciembre de 2018 Navarra Film Comisión ostenta el puesto como vocal dentro de la Junta Directiva de Spain Film Commissión. SFC es socio de EUFCN (European Film Commission Network) y AFCI (Association of Film Commissioners International), Institución con la que colabora de forma directa en la organización presencial y representativa de los diferentes festivales internacionales, juntas directivas y asambleas generales de las organizaciones en festivales como Berlinale, Cannes, MIPCOM O Ventana Sur de Argentina. Con especial dedicación en la industria de Bollywood, una de las más prolíficas a nivel mundial con el International Film Festival of India (IFFI); Film Bazaar (Goa; noviembre), Mumbai Film Festival (octubre) o el Diorama Film Festival (New Delhi; diciembre).

El I Encuentro Shooting in Spain, que cuenta con el patrocinio del Grupo Mediapro y el Grupo Secuoya y con la colaboración de la Secretaría de Estado de Turismo, 
ICEX - Invest in Spain, Consulado General de España de Los Ángeles, Gobierno de California y Alcaldía de Los Ángeles, tuvo como anfitrión al embajador honorario de Spain Film Commission, James Costos. Estaba previsto un encuentro en 2020 en España que ha tenido que ser trasladado a 2021, previsiblemente en primavera en el que se está trabajando activamente (http://www.shootinginspain.info/es/noticias-2/ primer-encuentro-virtual-shooting-in-spain-3-de-diciembre-2020).

Estas acciones se complementan con una actividad continua en reuniones y encuentros con todo tipo de stakeholders en materia de cine: Productoras, Consejo Navarro de Cultura, Departamentos de Gobierno de Navarra (Política Económica, Servicio Navarro de Empleo, Hacienda Foral de Navarra), Asociaciones Españolas de Animación (DIBOOS) y de Videojuegos (DEV), agentes locales (SODENA, Nasertic, UPNA, ATANA, Creanavarra), banca y asesorías fiscales, NAPAR, Cluster Audiovisual CLAVNA, empresas del sector.

La nueva reformulación del incentivo en 2020 ha permitido una mejor comunicación de las instituciones, gracias al cambio en el año 2019 del artículo 65.6 de la Ley Foral 26/2016 del impuesto sobre sociedades de Navarra, con la inclusión en la norma del uso del logotipo de Gobierno de Navarra y Navarra Film Comisión y la entrega de materiales, un claro y destacado posicionamiento y visibilidad de las instituciones.

Los datos que nos deja 2020 en comparativa con años anteriores de rodajes destaca que el número de producciones se ha mantenido pese a las dificultades:

\begin{tabular}{lrrrrc} 
Tipología & $\mathbf{2 0 1 6}$ & $\mathbf{2 0 1 7}$ & $\mathbf{2 0 1 8}$ & $\mathbf{2 0 1 9}$ & $\mathbf{2 0 2 0}$ \\
Largometraje ficción & 8 & 13 & 17 & 10 & 8 \\
\hline Largometraje documental & 9 & 4 & 11 & 6 & 11 \\
\hline Cortometraje & 20 & 20 & 25 & 47 & 32 \\
\hline Serie TV & 0 & 3 & 2 & 7 & 4 \\
\hline Spot & 15 & 16 & 18 & 14 & 13 \\
\hline Videoclip & 5 & 6 & 5 & 3 & 6 \\
\hline Programa TV & 9 & 10 & 11 & 12 & 6 \\
\hline Video institucional/ Rp. foto & 1 & 4 & 6 & 0 & 16 \\
\hline Total & $\mathbf{6 7}$ & $\mathbf{7 6}$ & $\mathbf{9 5}$ & $\mathbf{9 9}$ & $\mathbf{9 6}$ \\
\hline
\end{tabular}

\section{CLÚSTER AUDIOVISUAL DE NAVARRA CLAVNA}

El Clúster tuvo once nuevas adhesiones. Se puede destacar la capacidad asociativa en tiempos de incertidumbre.

La asociación agrupa al sector y presentó, tal y como se destaca al inicio de este artículo, la solicitud de medidas urgentes para la reactivación. 
Impulsa el portal online Navarra Film Industry (navarrafilmindustry.com), que se ha consolidado en 2020, donde se recoge toda la información de interés de la industria. Ha actualizado la Guía Audiovisual de Navarra referencia para productores y profesionales.

La búsqueda de proyectos y financiación es evidente con la concesión de 1,4 millones de euros al proyecto de $\mathrm{I}+\mathrm{D}$ «Emotional films» -que va a pasar a llamarse «emotional art»-. Se trata del primer proyecto de I+D del sector audiovisual que recibe una subvención de esa cuantía por parte del Gobierno de Navarra, lo que hace consolidarse al sector como estratégico, también en el I+D.

El acuerdo Valonia-Navarra se presentó en un plató virtual y abrió el camino para el desarrollo de esta herramienta por parte del clúster, para ofrecer este servicio de realidad virtual al tejido industrial y empresarial navarro. Va a permitir el desarrollo de la industria de la animación en nuestra región, así como su propia internacionalización. SODENA y el fondo belga Wallimage colaboran por la industria audiovisual. El presidente de la sociedad pública navarra, Manu Ayerdi, firmó en noviembre el memorando de colaboración con el fondo audiovisual Wallimage de Valonia (Bélgica) para promover la industria audiovisual en ambas regiones. Durante tres años, ambas partes compartirán experiencias con el objetivo de sentar las bases para coproducciones audiovisuales, formular herramientas de financiación de la animación o desarrollar la formación para profesionales del sector, entre otras acciones.

La formación se ha realizado junto con el Servicio Navarro de Empleo. Se organizaron cursos de oficios de cine impartidos por profesionales en activo y con larga trayectoria, formando en el departamento de arte, producción, fotografía y postproducción a más de cuarenta alumnos, con más de ciento cincuenta inscripciones.

\section{FILMOTECA DE NAVARRA}

La Filmoteca de Navarra a lo largo del año 2020 ha seguido dando continuidad a los criterios de programación y actividades que se establecieron desde su inicio, alimentando la sala de proyecciones de la Biblioteca y Filmoteca de Navarra, las cuales, han tenido lugar, al menos los miércoles, jueves y viernes laborables, de enero a marzo y de septiembre a diciembre, a razón de una sesión diaria -pese a las restricciones, 99 sesiones, exactamente las que corresponderían a un año habitual, deduciendo las que no se pudieron celebrar en abril, mayo, junio y la mitad de marzo-.

Los criterios de programación han estado orientados a la diversidad, con la difusión del hecho cinematográfico como valor cultural, con referentes esenciales como la cita con el cine clásico, la muestra del cine de otras regiones del mundo, el espacio obligado para la difusión de la creación audiovisual local, el esencial cine silente (con varias sesiones acompañadas por música en vivo), el cine contemporáneo, la colaboración con otras entidades en ciclos específicos y la versión original subtitulada, como muestra de respeto a la creación original. Se revisan todas y cada una de las copias a proyectar, con el fin de que las sesiones públicas estén al nivel de lo que se debe exigir a una filmoteca. 


\subsection{Ciclos básicos}

La mayor parte de los ciclos que se realizan en la Filmoteca de Navarra tienen una duración de un mes. Las dos únicas muestras que superan las cuatro o cinco sesiones son los ciclos de programación propia denominados Cine imprescindible y «esencial» (Luchino Visconti esencial).

Como programaciones asentadas en la parrilla de la filmoteca encontramos: Cine Imprescindible (programa propio de la Filmoteca de Navarra, dedicado a la recuperación de filmes esenciales de la Historia del cine, ordenados por cronología), cine «esencial» (programación propia dedicada a un cineasta relevante, concretada en diez títulos), al igual que la llamada Foco Punto de Vista, otra cita, mensual en este caso, que concreta la presencia a lo largo del año del Festival de Cine Documental, el rincón Filmoteca navarra, que ha incorporado en 2020 ya de modo sistemático la sección Generazinema, dedicada al mostrar los filmes subvencionados por el Gobierno de Navarra.

La interrupción de la programación en marzo, impidió la celebración de ciclos ya diseñados como el que habitualmente se coordina con Nosferatu (Patronato de Cultura de San Sebastián, e IVAC de Valencia), el veterano Arquitectura de cine, que se celebra en el mes de mayo, un ciclo dedicado al Camino de Santiago, otro dedicado a Eric Rohmer, el confeccionado con el Colegio de Abogados «La abogacía en el cine». En el ámbito de la muestra de cinematografías del resto del mundo tuvimos la ocasión de llevar nuestra mirada al cine polaco (marzo), palestino (octubre), cine hindú (septiembre), o al cine africano (noviembre).

En el año 2020 se ha celebrado la VII edición del Roof Top Cinema en la terraza de Baluarte. Se aumentaron de tres a cinco las sesiones, para "compensar»la ausencia de la Filmoteca en la palestra, centrando el tema del evento en historias de la terraza "Rooftop Stories». La última sesión, On the Roof, estreno en España de este filme checo, estuvo presentada por el director del Centro Checo en España, Stanilsav Skoda. Esta programación hace visible la labor de la Filmoteca fuera de su propia sede.

La Filmoteca ha vuelto a participar en la organización de Pamplona Negra, una cita con el género negro en el cual es inevitable la referencia al cine. En esta quinta edición «El lado más oscuro", se vieron las siguientes películas: Sed de mal (O. Welles, 1958), El detective (G. Douglas, 1968), Serpico (S. Lumet, 1973) y L. A. Confidential (C. Hanson, 1997), todas ellas orientadas a diseccionar el asunto de la corrupción policial.

\subsection{Colaboraciones con otras instituciones}

Cada vez son más los colectivos, grupos u otras entidades, las que solicitan albergar sus programas en la Filmoteca: Escuela Oficial de Idiomas de Pamplona, Festival Internacional de Cine Documental de Navarra Punto de Vista, Filmoteca Española, 
Museo de Navarra, Biblioteca de Navarra, Departamento de Políticas Migratorias y Justicia del Gobierno de Navarra, Noucinemart, África es imprescindible, Ateneo Navarro/Nafar Ateneoa, NAPAR, CLAVNA, Universidad Pública de Navarra, Instituto Polaco de Cultura, Centro Checo de Madrid, F. W. Murnau Institut, The British Council, Pasarela 948, Festival Rizoma, Beltza Weekend, Asamblea de Cooperación por la Paz, Museo del Carlismo de Navarra, Amnistía Internacional, SodePaz.

\subsection{Año especial}

La suspensión de las sesiones el 14 de marzo, impulsó al Área de Programación a mantener activa la vinculación con el público cinéfilo. Se organizaron dos concursos de preguntas/respuestas, con periodicidad diaria, a lo largo de los meses de abril, mayo y junio. También se organizó un concurso de dibujos de cine, para los más pequeños que posteriormente fueron expuestos en la Filmoteca, con premios a los ganadores con libros y películas de video.

Se lanzó una colección de libros de cine, con el fin principal de reconocer la actividad de numerosos navarros en el ámbito cinematográfico, especialmente aquellos que nunca han sido biografiados. Se editó en el mes de enero un libro dedicado a la actriz Camino Garrigó y en el mes de diciembre el n. ${ }^{\circ} 2$ de la colección, dedicado al actor Joaquín F. Roa.

Los precios de las sesiones se han mantenido ( $3 €$ precio general; $1 €$ para sesiones de «Filmoteca Navarra»; entrada libre, cuando los titulares de derechos así lo exigen; abono de diez sesiones: $20 €$ ). El número de sesiones con que terminó el año fue de 99, y los espectadores 8300, aproximadamente. La media de espectadores por sesión cerrará en torno a 83, equivalente a un índice de ocupación del $81 \%$. Hay que tener presente para evaluar estos datos, que entre el 1 de septiembre y el 31 de diciembre, el aforo de la sala ha oscilado entre el $50 \%$ (88 butacas) y el $30 \%$ (52 butacas), pasando por el $40 \%$ (70 butacas).

Se puede destacar el claro objetivo de normalización: salvo las restricciones de aforo y las medidas de seguridad exigibles, la actividad se ha mantenido con total ausencia de incidencias, contribuyendo a la normalización de la actividad cinematográfica.

\section{FESTIVAL PUNTO DE VISTA}

Más de mil inscripciones fueron enviadas para participar en la decimocuarta edición del Festival Punto de Vista. Solo veintitrés películas de quince países diferentes conformaron la Sección Oficial, cinco largometrajes y dieciocho cortos.

Algunas de las exploraciones temáticas fueron la búsqueda de los orígenes y la identidad por parte de cineastas jóvenes que reconstruyen su historia familiar, las voces feministas desde el trasvase generacional y creativo, la reflexión sobre la herencia materna y las reivindicaciones ecologistas con perspectiva feminista, con especial aten- 
ción a la observación de los tiempos y dinámicas de la naturaleza. Tal y como recogía la memoria del Festival.

El palmarés fue variado y en su conjunto un viaje por el cine experimental del mundo. Gran Premio Punto de Vista: ex aequo, Un film dramatique, de Eric Baudelaire, 2019, Francia, y Apiyemiyekî, de Ana Vaz, 2019, Brasil, Francia, Portugal, Países Bajos. Premio Jean Vigo a la Mejor Dirección: Once Removed, de Lawrence Abu Hamdan, 2019, Líbano. Premio al Mejor Cortometraje: Now, at last!, de Ben Rivers, 2019, Brasil, Reino Unido. Mención Especial del Jurado: Queen, de Kathryn Elkin, 2019, Reino Unido y Aquí y allá, de Melisa Liebenthal, 2020, Argentina, Francia. Premio Especial del Público a la Mejor Película: Overseas, de Sung-A Yoon, 2019, Bélgica, Francia. Y el Premio de la Juventud para Queen, de Kathryn Elkin, 2019, Reino Unido.

\section{LO QUE VIENE}

El certamen de cine y series «Lo que viene» que organiza la Asociación de Informadores Cinematográficos de España, alberga presentaciones de próximos proyectos de las grandes productoras españolas y celebró un ciclo de preestrenos que disfrutó el público de Tudela al aire libre las noches del 15, 16 y 17 de julio:

- Las actrices Macarena Gómez y Lorena López, el codirector Juanjo Moscardó y la guionista María Mínguez, presentaron su película Amor en polvo, a espectadores y prensa.

- El director de Blanco en blanco, el hispano-chileno Théo Court, también compartió su último trabajo, que fue galardonado con dos premios en el Festival de Venecia y con el FIPRESCI en La Habana en 2019.

- Patricia López Arnaiz y David Ilundain, actriz y director de Uno para todos.

- Buena parte del equipo artístico de la serie Albanta de Flooxer, protagonizada por Kimberley Tell, Pol Monen, Lucía Martín, Eva Llorach, Pep Llopis, Javier Ruesga y Jorge Clemente.

Estuvieron presentes la directora del ICAA, Beatriz Navas; el director del Festival de San Sebastián, José Luis Rebordinos; del Festival de Sevilla, José Luis Cienfuegos; del Festival D’A, Carlos R. Ríos; y contó también con el presidente de AISGE y Premio Feroz de Honor 2020, Emilio Gutiérrez Caba. Participaron en mesas redondas productoras como A Contracorriente, Filmin, BTeam, Avalon, Vaca Films, Morena Films, Grup Balañá, La Aventura Audiovisual e instituciones como la Navarra Film Comission, la Consejería de Cultura de Navarra, el Cluster Audiovisual de Navarra.

Se pudo participar en charlas gratuitas, previa inscripción en www.loqueviene.es, promovidas por el Consorcio EDER.

Lo que viene cuenta con el patrocinio del Gobierno de Navarra, Turismo de Navarra, la Comunidad de Bardenas Reales y el Grupo Enhol y con la colaboración del 
Ayuntamiento de Tudela, el Ayuntamiento de Corella, La Escuela de Arte y Superior de Diseño de Corella y el Ministerio de Cultura y Deporte.

\section{CONECTA FICTION}

Conecta Fiction, el evento de coproducción para series celebrado entre el 15 y el 18 de junio, cerró su actividad con cuatrocientos participantes, indicativo del momento que viven las series. El tema central de los paneles fue el de reinicio de talento con talleres on line para diálogo y interacción entre usuarios. Los países con mayor participación fueron España, Francia y Reino Unido así como Estados Unidos, México y Argentina.

La cuota más alta la tuvo la inauguración donde se presentaron primeras imágenes de la serie nacida en Conecta "Inés del alma mia» con 1460 usuarios únicos. También, la presentación en abierto de la serie Antidisturbios de Movistar.

\section{SALAS COMERCIALES}

Las salas de cine se mantuvieron cerradas por la falta de estrenos durante meses muy importantes de negocio. Con el paso a la fase 2 de la desescalada y durante todo el año, las salas de cine abrieron sus puertas al público con un aforo limitado al $30 \%$ de sus butacas y con toque de queda que cancelaba la sesión de noche, una de las más compradas por el público. Sin embargo, la escasez de oferta de películas ha llevado a las empresas exhibidoras a posponer su reapertura hasta completar una programación que atraiga a los espectadores. Proyectos como el autocine en el Navarra Arena, promovido por Cines Golem, ayudaron a la promoción y reinvención.

\section{PUNTO Y SEGUIDO}

Afortunado el sector audiovisual que ha sabido mantener la cabeza fuera del agua. Herencia del trabajo asociativo, las reivindicaciones industriales y el saber reinventarse a tiempo-online, autocine, acuerdos internacionales-. Los profesionales y técnicos autónomos del sector son los que más han sufrido, carentes de subcontratas y proyectos-motor a los que subirse, muchos de ellos acogidos a numerosos ERTE, con ayudas muy escasas para solventar los gastos fijos, costes e impuestos, que cabría llevarlos a revisables. Un sector que gracias al incentivo sigue siendo atractivo como territorio de rodaje y producción de películas y series como fueron Ana Tramel con Maribel Verdú, La pasajera de Fernando González y Raúl Cerezo o Dos de Mar Targarona. Y donde los Goya tuvieron representación navarra en nominados: Raúl de la Fuente y Amaia Remírez optaron al Mejor Cortometraje Documental por El Infierno y Mikel Serrano Lecea a la mejor dirección artística, casi como una premonición para el Goya 2021.

Mantenida la promesa de solo aparecer una vez Covid-19, con nombre de película de ciencia ficción, de tarde de domingo en familia. El 2020 estuvo lleno de domingos. 


\section{LISTA DE REFERENCIAS}

La información para la elaboración del artículo se ha obtenido también de las siguientes personas y entidades:

- Diario de Noticias de Navarra.

- Diario de Navarra.

- Todonews.com.

- Clavna.com.

- Quijote.tv.

- Alberto Cañada, director de Programación, Filmoteca de Navarra/Nafarroako Filmoteka.

- Sara Sevilla, directora, Navarra Film Commission (NFC).

- Quim Torrents y María Guzmán, NAPAR, Asociación de productoras de Navarra.

- Silvia Cañada y Arturo Cisneros, Clúster Audiovisual de Navarra (CLAVNA).

- Javier Lacunza, gerente, Navarra de infraestructuras de Cultura, Deporte y Ocio (NICDO). 